\title{
Bond Strength and Water Resistance of a Resin Composite Bonded to Glass Plates Treated with Commercial Ceramic Primers
}

\author{
Katsura OHASHI ${ }^{1,2}$, Kaori MIYAKE ${ }^{1,2}$, Tota SHIMIZU ${ }^{3}$, Akinori OHNO ${ }^{3}$, Toru SHIIYA ${ }^{2,4}$, Yoshiharu \\ MUKAI $^{2,4}$, Toshiyuki SUZUKI ${ }^{3}$, Ayako TERANAKA ${ }^{5}$, Hirotoshi IWAI ${ }^{5}$, Satoshi HIRAYAMA ${ }^{5}$, Katsuhiko \\ KIMOTO $^{3}$ and Tomotaro NIHEI ${ }^{1,2}$ \\ 1. Division of Clinical Biomaterials, Department of Oral Science, Graduate School of Dentistry, Kanagawa Dental University, \\ Yokosuka, Kanagawa 238-8580, Japan \\ 2. Institute of Oral Regenerative Medicine, Kanagawa Dental University, 82, Inaoka-cho, Yokosuka, Kanagawa 238-8580, Japan \\ 3. Division of Prosthodontics and Oral Implantology, Dentistry for Oral Interdisciplinary Medicine, Graduate School of Dentistry, \\ Kanagawa Dental University, Yokosuka, Kanagawa 238-8580, Japan \\ 4. Division of Cariology and Restorative Dentistry, Department of Oral Interdisciplinary Medicine, Graduate School of Dentistry, \\ Kanagawa Dental University, Yokosuka, Kanagawa 238-8580, Japan \\ 5. Department of Operative Dentistry, School of Dentistry at Matsudo, Nihon University, Matsudo, Chiba 271-8587, Japan
}

\begin{abstract}
Background: Silane coupling agents are used as a bonding agent between ceramics and resins in dentistry. The effectiveness of these agents is often enhanced by the addition of acid or by heat treatment. Objective: This study aimed to evaluate, the clinical characteristics, TBS (tensile bond strength) and water resistance of eight commercial ceramic primers (seven silane coupling agents and one alumina zirconia primer). Method: Glass plates were used as the adherent. The TBS of the resin composite and the wettability of the resin monomer to glass surfaces treated with ceramic primers was investigated, with 3-MPS (methacryloxypropyltrimethoxysilane) used as a control. The values obtained from the experiments were analyzed using one-way ANOVA followed by Tukey's multiple comparison tests $(p<0.05)$. Results: No significant differences in TBS were observed between the commercial products and MPS, except for the alumina zirconia primer. However, four products (three of the seven agents and the alumina zirconia primer) exhibited significantly lower TBS values after application of thermal stress when compared with those stored in water $(p<0.05)$. All but one of the eight primers displayed significantly higher contact angles between the treated glass and the resin monomer when compared with MPS $(p<0.05)$. These results suggest that some commercial ceramic primers may contribute to increased strength and durability.
\end{abstract}

Key words: Silane coupling agent, resin composite, bond strength, bond durability, water resistance, wettability.

\section{Introduction}

The use of silane coupling agents for the bonding of organic and inorganic substrates is standard practice. In dentistry, silane coupling agents are used as a bonding agent between ceramics and resins, and as a surface treatment for filler particles in resin composites [1-6]. Several commercially available

Corresponding author: Tomotaro NIHEI, professor, research field: dental materials. dental ceramic primers contain 3-MPS (methacryloxypropyltrimethoxysilane) as the primary active component. The effectiveness of silane coupling agents is often enhanced by the addition of acid or by heat treatment. However, breakage of siloxane bonds is known to occur after this treatment; the resulting hydrolysis is caused by occlusal stress and thermal or chemical stimulation during food consumption [7-15]. 
Previously, we evaluated the water resistance of silane coupling agents by examining the bond strength between resin composite and ceramics or fused silica glass treated with silane coupling agents containing hydrophobic fluorocarbon chains or a phenyl group; significantly higher bond strength and water resistance were observed relative to the samples treated with MPS alone [16-18]. Subsequently, we investigated the bond strength between resin composites and glass surfaces treated with MPS after addition of acid to evaluate the catalytic effect that is known to improve the condensation reaction of silane coupling agents [19]. The use of $5.0 \mathrm{vol} \%$ of phosphoric acid resulted in significantly higher bond strength, which was maintained even after thermal stress, indicating the effectiveness of catalyst in improving the activity of the silane coupling agent.

The various forms of dental ceramic primers, include a two-bottle type mixed immediately before application, a one-bottle type with a catalyst, and one that requires acid treatment, all of which contain some type of catalyst.

In the present study, the bond strength of resin composite and the wettability of resin monomer to glass surfaces treated with commercially available ceramic primers were investigated in order to evaluate their clinical characteristics and water resistance and to further understand the efficacy and function of the primers.

\section{Materials and Methods}

\subsection{Materials}

Details concerning the eight commercial ceramic primers (seven silane coupling agents and one alumina zirconia primer) used in this study are presented in Table 1. Primers were used according to the manufacturers' instructions as received. MPS (Lot \# 206886, Shin-Etsu Chemical, Tokyo, Japan) was prepared as a $50 \mathrm{mmol} / \mathrm{L}$ ethanol solution (Wako Pure Chemistry, Osaka, Japan), with 5 vol\% phosphoric acid composing 10 vol $\%$ of the solution added immediately before application to the glass surface.

\subsection{Tensile Bond Strength Tests}

Glass plates $(15 \times 15 \times 3 \mathrm{~mm}$, Asahi Glass Co., Tokyo, Japan) were used as the adherend. To remove any contamination from the surface, the glass plates were first soaked in $1 \mathrm{~mol} / \mathrm{L}$ sodium hydroxide solution (Wako Pure Chemistry, Osaka, Japan) for one day, and in $1 \mathrm{~mol} / \mathrm{L}$ hydrochloric acid solution (Wako Pure Chemistry, Osaka, Japan) the following day. They were then thoroughly rinsed with distilled water, and dried at $50{ }^{\circ} \mathrm{C}$ for 2 hours. One surface of the glass plates was treated with each of the eight primers according to the manufacturers' instructions

Table 1 Contents of ceramic primers used in this study.

\begin{tabular}{|c|c|c|c|c|c|}
\hline Material & Manufacture & Code & \multicolumn{2}{|l|}{ Composition } & Contents \\
\hline Porcelain Primer & Shofu & IN & \multicolumn{2}{|l|}{ one-bottle } & silane coupling agent, absolute ethanol, etc. \\
\hline Monobond S & Ivoclar Vivadent & MO & \multicolumn{2}{|l|}{ one-bottle } & $\begin{array}{l}\text { silane coupling agent }(\gamma \text {-MPTS }) \text {, ethanol, } \\
\text { water, acetic acid }\end{array}$ \\
\hline \multirow{2}{*}{ Porcelain Liner M } & \multirow{2}{*}{ Sun Medical } & \multirow{2}{*}{ PL } & \multirow{2}{*}{ two-bottles } & liquid $\mathrm{A}$ & MMA, 4-META, etc. \\
\hline & & & & liquid B & MMA, silane coupling agent $(\gamma$-MPTS $)$, etc. \\
\hline RelyX ${ }^{\mathrm{TM}}$ Ceramic Primer & $3 \mathrm{M}$ ESPE & RE & \multicolumn{2}{|l|}{ one-bottle } & silane coupling agent, ethanol, water \\
\hline \multirow{2}{*}{ Ceramic Primer } & \multirow{2}{*}{$\mathrm{GC}$} & \multirow{2}{*}{$\mathrm{CP}$} & \multirow{2}{*}{ two-bottles } & primer A & vinyl silane, ethanol \\
\hline & & & & primer B & methacrylic acid ester, ethanol \\
\hline \multirow{2}{*}{ Tokuso Ceramic Primer } & \multirow{2}{*}{ Tokuyama Dental } & \multirow{2}{*}{ TO } & \multirow{2}{*}{ two-bottles } & liquid $\mathrm{A}$ & silane coupling agent, ethanol \\
\hline & & & & liquid B & monomer of phosphoric acid, ethanol \\
\hline Clearfil $^{\circledR}$ Ceramic Primer & $\begin{array}{l}\text { Kuraray Noritake } \\
\text { Dental }\end{array}$ & $\mathrm{CS}$ & \multicolumn{2}{|l|}{ one-bottle } & silane coupling agent, MDP, ethanol \\
\hline AZ Primer & Shofu & $A Z$ & \multicolumn{2}{|l|}{ one-bottle } & $\begin{array}{l}\text { 6-methacryloxy hexyl phosphonoacetate, } \\
\text { aceton, etc. }\end{array}$ \\
\hline
\end{tabular}


using microbrushes (Shofu, Kyoto, Japan). A layer of MPS was applied, and the glass plates were left to dry at room temperature for 5 minutes. An MPS-treated glass plate (no other primer) was used as a positive control, whereas untreated silane (no primer) was used as the negative control. The treated surface of the glass plate was covered with mending tape $\left(\operatorname{Scotch}^{\mathbb{B}}\right.$ Mending Tape 810, Sumitomo 3M, Tokyo, Japan) with a round perforation ( $3 \mathrm{~mm}$ diameter) to define the bonded surface. Resin composite (Clearfil ${ }^{\mathrm{TM}}$ FII, Paste A and B: Lot \# 00061A and 00034A, Kuraray-Noritake, Tokyo, Japan) was mixed according to the manufacturer's instructions and placed on the bonding surface of a mesh type stainless steel rod $5 \mathrm{~mm}$ in diameter (Tomy International, Tokyo, Japan). The glass surface and the rod were manually held together for a few minutes to achieve bonding after which the specimens were left in the air at room temperature for 30 minutes. Ten samples of each primer and the positive and negative controls were prepared, and divided into two different immersion groups. Half of the samples were stored in distilled water at $37{ }^{\circ} \mathrm{C}$ for 7 days (DW group), and the other group was subjected to thermal cycling (4-60 ${ }^{\circ} \mathrm{C}, 40$-second dwell time, $\left.\times 10,000\right)$ after storage in distilled water at $37{ }^{\circ} \mathrm{C}$ for 1 day (TC group). The tensile bond strength of each sample was measured using an Autograph (AGS-500, Shimadzu, Kyoto, Japan) at a crosshead speed of $1 \mathrm{~mm} / \mathrm{min}$.

\subsection{Contact Angles of Resin Monomer on Treated Surface}

Resin monomer prepared by mixing bisphenol A-glycidyl methacrylate (bis-GMA, Lot \# 11-29-17, Monomer-Polymer \& Dajaac Labs, Trevose, PA, USA) and triethyleneglycol-dimethacrylate (TEGDMA, Lot \# GD01, Tokyo Chemical Industry, Tokyo, Japan; weight ratio of 1:1) was dropped onto the treated glass plates. The contact angles for each sample were obtained using an automatic contact angle-measuring device (DCA-VZ, Kyowa Interface
Science, Saitama, Japan).

\subsection{Statistical Analysis}

The values obtained from the experiments were analyzed using one-way ANOVA followed by Tukey's multiple comparison test $(p<0.05)$.

\section{Results}

\subsection{Tensile Bond Strength}

The tensile bond strength between resin composite and treated glass plate for each sample is shown in Fig. 1. Fig. 2 illustrates the type of bond failure at the fracture surface after tensile testing.

The bond strength of the MPS alone was $13 \mathrm{MPa}$ after water immersion with no significant decrease after application of thermal stress ( $9 \mathrm{MPa} ; p>0.05)$. The MPS control specimens demonstrated cohesive failure of the resin composite or fracture of the glass after tensile testing; however, the occurrence of complex failures was more common under thermal stress. As expected, the bond strength of the negative control was $4 \mathrm{MPa}$, which was significantly lower than that of MPS $(p<0.05)$. It was not possible to measure the bond strength of the negative control after thermal stress owing to the detachment of all the stainless steel rods from the glass surface (adhesive failure) during the experiment.

The bond strengths of all of the ceramic primers (except for alumina zirconia, AZ) were above $10 \mathrm{MPa}$, after water storage, showing no significant difference from that of MPS alone $(p>0.05)$. No significant decrease in bond strength after thermal stress was observed when compared to the water storage group $(p>0.05)$ for IN, MO, PL and RE. Nevertheless, the values for $\mathrm{CP}$, TO and $\mathrm{CS}$ were lower than $6 \mathrm{MPa}$ after thermal stress, which is a significant decrease when compared with the water storage samples $(p<$ $0.05)$. The bond strength of the AZ primer after water storage was significantly lower than MPS $(p<0.05)$ at $4 \mathrm{MPa}$; the value after thermal stress could not be 


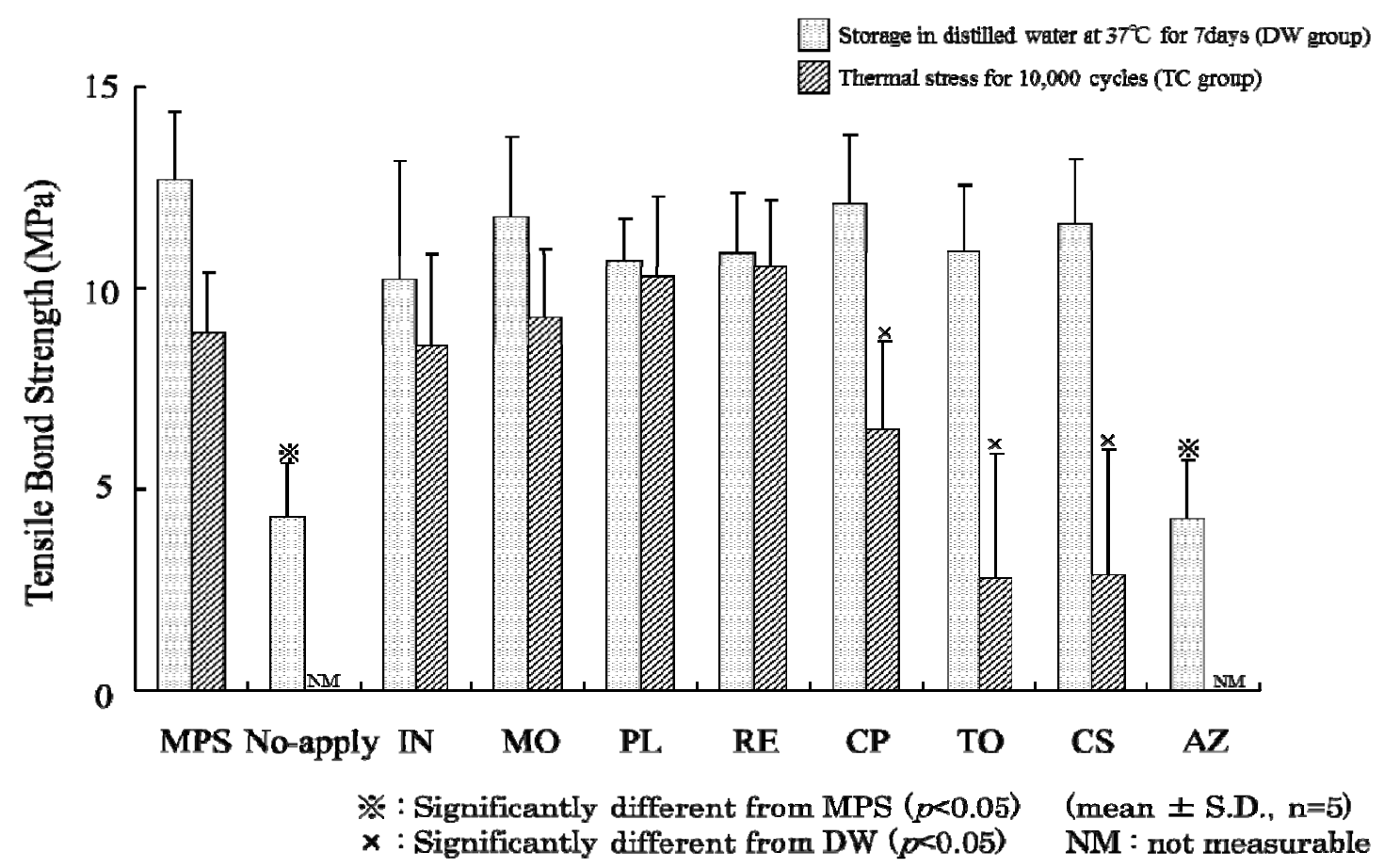

Fig. 1 Tensile bond strength of resin to glass plates treated with each ceramic primers. A positive control group is MPS (3-methacryloxypropyltrimethoxysilane), a negative control group is no-apply (no silane treatment).

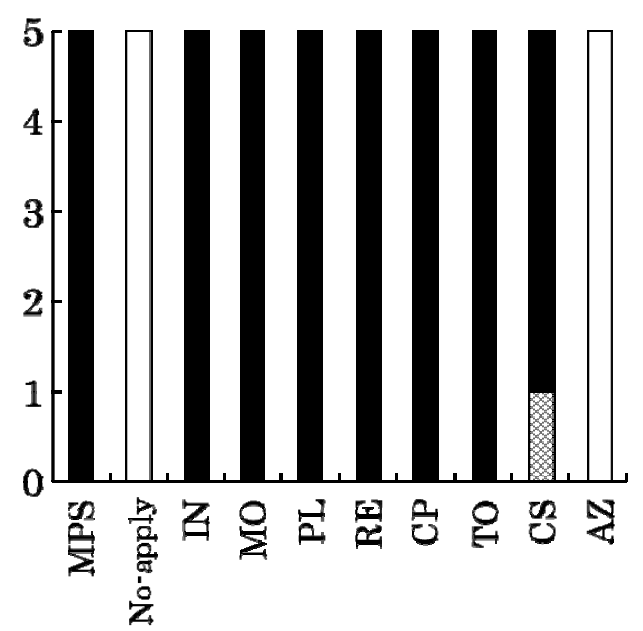

Stored in distilled water at $37^{\circ} \mathrm{C}$ for 7 days

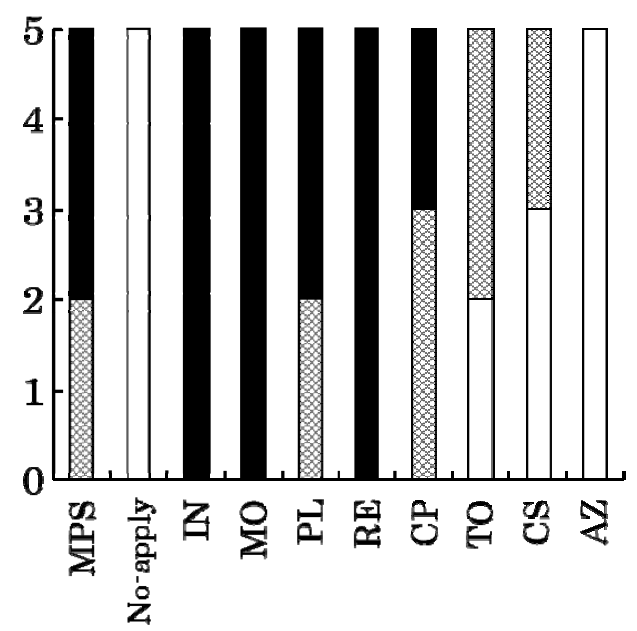

Thermal stress for 10,000 cycles

$\square$ adhesive failure, 图 complex type,

"cohesive failure" or "glass fracture"

Fig. 2 The types of fractured surface after tensile bond test. No-apply and AZ groups were adhesive failure in all specimens, and the other groups were increased more common complex failure after thermal stressed.

measured due to adhesive failure.

All samples primarily demonstrated either cohesive failure in the resin composite or glass fracture on the fractured surface of the commercial silane coupling agent after tensile testing. On the other hand, after thermal stress, there was an increased incidence of complex or adhesive failures between the resin composite and the glass surface. Following thermal stress application, the IN, MO and RE specimens exhibited cohesive failures of the resin composite or glass fractures, whereas the TO and CS specimens presented with increased adhesive failures between the 
resin composite and glass surface. All AZ specimens underwent adhesive failure between the resin composite and glass surface in both DW and TC groups.

\subsection{Wettability of the Resin Monomer on Treated Glass Plates}

The contact angles of the resin monomer applied to the treated and untreated glass plates are displayed in Table 2. As expected, the contact angle of the negative control $\left(34.8^{\circ}\right)$ was significantly higher than that of MPS $\left(20.8^{\circ} ; p<0.05\right)$. The contact angles of PL and AZ were approximately $21^{\circ}$, not significantly different from MPS $(p>0.05)$. However, in the remaining six commercial ceramic primers, the contact angles ranged between $23^{\circ}$ and $40^{\circ}$, and were significantly higher than that of MPS $(p<0.05)$.

\section{Discussion}

Silane coupling agents are silicon-based chemicals that contain organic substances. In general, they are used as coupling agents to facilitate the adhesion of organic and inorganic materials. In dentistry, silane coupling agents are indispensable in various clinical situations; for example, during the use of resin composites as restorative materials, silane coupling agents are utilized as surface treatments for the filler particles [1]. In addition, they are used for the bonding of glass ceramic restorations, the repair of crowns with resin facings or as a pretreatment material in resin crowns. However, the surface layer treated with silane coupling agents is subjected to thermal changes, water contamination, and intermittent mechanical stress due to eating, brushing, and simple occlusion. The harsh environment of the oral cavity is thought to cause deterioration in the physical properties of the treated layers over time [7-15].

In the present study, the clinical characteristics including the hydrolytic stability of commercially available ceramic primers were evaluated in terms of bond strength and wettability. As shown in previous studies [17, 19], MPS undergoes a decrease in tensile bond strength after thermal stress relative to water storage, but the difference observed was not significant $(p>0.05)$. On the other hand, the proportion of complex failures during tensile bond test increased significantly after thermal stress suggesting that the siloxane bond in the silane-treated layer was broken due to thermal stress, resulting in a decrease in bond strength and an increase in complex failures at the fracture surface. Since chemical bonding was not accomplished between the resin composite and the glass surface in the negative control, the bond strength after DW storage was significantly lower than that seen in MPS $(p<0.05)$. The stainless steel rods had detached from the glass surface during TC storage. Moreover, all negative control specimens exhibited adhesive failure after DW storage and TC storage.

For the AZ primer, similar trends to the negative control were found with respect to tensile bond strength and fracture type after bond strength testing. This may be attributed to the fact that the AZ primer does not contain a silane coupling agent, and therefore, similar to the negative control, no chemical bonding was established. On the other hand, the seven commercial ceramic primers exhibited tensile strengths greater than $10 \mathrm{MPa}$ after DW storage, though not significantly different from MPS. All except for CS presented with cohesive failure in the resin composite or glass fracture after tensile bond strength testing. The manufacturers did not disclose the concentrations of the silane coupling agents (although AZ contains

Table 2 Contact angles of resin monomer on treated glass plates.

\begin{tabular}{|c|c|c|c|c|c|c|c|c|c|c|}
\hline & MPS & No-apply & $\mathrm{IN}$ & $\mathrm{MO}$ & PL & $\mathrm{RE}$ & $\mathrm{CP}$ & TO & $\mathrm{CS}$ & $\mathrm{AZ}$ \\
\hline Contact angle $\left(^{\circ}\right)$ & 20.8 & $34.8^{*}$ & $25.8^{*}$ & $30.0 *$ & 20.8 & $27.8^{*}$ & $39.3 *$ & $23.2 *$ & $25.3^{*}$ & 21.4 \\
\hline S.D. & 0.5 & 1.3 & 0.3 & 0.9 & 0.5 & 0.6 & 0.7 & 0.8 & 0.4 & 0.8 \\
\hline
\end{tabular}

* Significantly different from MPS $(p<0.05)$. 
$0 \%$; Table 1). Clearly, they have developed their products taking stability and adhesiveness into account, and the bond strength of their products is comparable to MPS alone as a positive control. However, the tensile bond strengths of IN, MO, PL and RE, after TC storage, were not significantly decreased relative to the values obtained after DW storage, demonstrating a higher level of bond strength than MPS alone. Although some PL specimens displayed increased complex failure after tensile testing, the others (IN, MO and RE) exhibited either cohesive failure in the resin composite or glass fracture. The tensile bond strengths of $\mathrm{CP}, \mathrm{TO}$ and $\mathrm{CS}$ after TC storage, were significantly lower than the values obtained after water storage $(p<0.05)$ with an increase in the proportion of complex or adhesive failures. In addition, it was difficult to determine any trends based on primer composition. It has been reported that silane coupling agents, especially the one-bottle types, tend to lose their clinical efficacy over time due to a condensation reaction, a recognized behavior in silanes [20]. However, the ceramic primers used were all freshly manufactured, and no differences in water resistance in terms of bond strength were noted between the one-bottle and two-bottle types. We believe that the results of the afore-mentioned study might have been different if older samples of commercial ceramic primers had

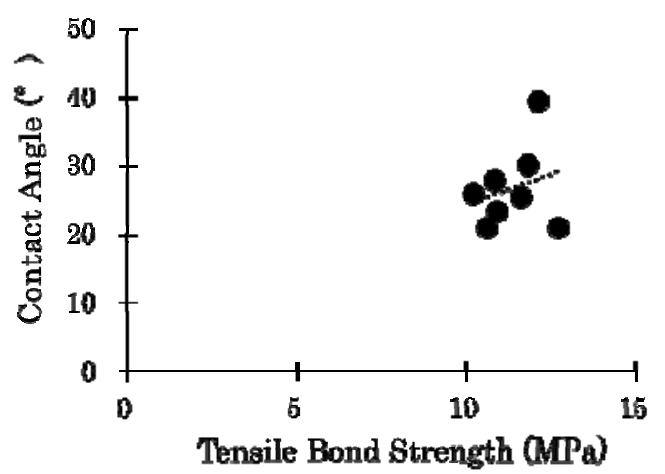

Storage in distilled water at $37^{\circ} \mathrm{C}$ for 7 days been used.

The negative control had a significantly higher contact angle between the glass surface and the resin monomer when compared with MPS $(p<0.05)$. There was no correlation between wettability and bond strength in the commercial ceramic primers (Fig. 3). Yamaguchi [21] reported that bond strength was improved by low contact angles with high wettability, but the results of the present study do not support these findings. It can be assumed that additives and variations in concentration of the commercial ceramic primers used may have affected the results.

Meng et al. [22] reported no correlation between the silane concentration and the siloxane quantity of commercial silanes, as analyzed by FTIR. They reported that silane activation and condensation are affected not only by silane concentration, but also by the solvent. Moreover, they reported a negative correlation between the contact angle of the bonding agent to a silanized ceramic surface and bond durability, and no correlation between siloxane quantity and bond durability. Thus, it was suggested that commercial ceramic primers possess the required amounts of bond durability and wettability for a resin monomer due to the variety of silane components.

We have previously reported that the water resistance of a silane coupling layer is affected by cross-linking in the silane layer, owing to differences

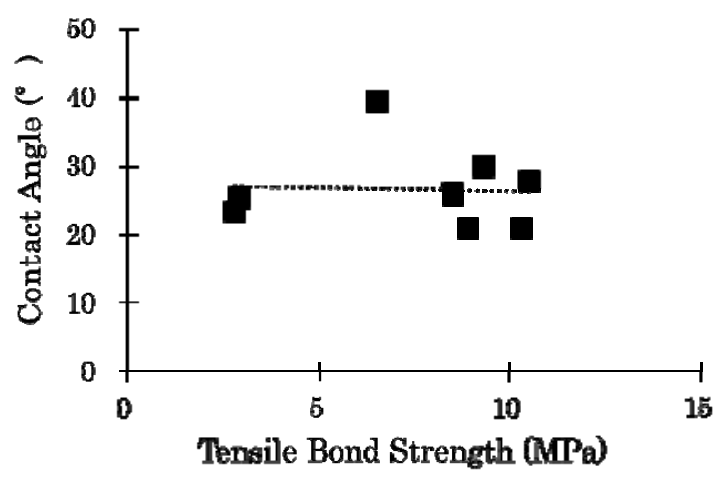

Thermal stress for 10,000 cycles

Fig. 3 Correlation diagrams of tensile bond strength and contact angle. There was no correlation between wettability and bond strength in the ceramic primers. 
in the hydrolyzable groups of various silanes [17]. The results of this study suggest differences in the cross-linking of commercial ceramic primers. In previous studies, the hydrolysis reactions of single-bottle silane have been shown to be superior to that of the two-bottle silane coupling agents; on the other hand, two-bottle silanes was higher shown to be higher durability [23]. However, the findings of the present study are not in accordance with these results. No differences in bond strength, durability or wettability were observed between the single-bottle and two-bottle primers in this study.

The results presented herein demonstrate that there is no significant difference in bond strength among commercial ceramic primers, but there is a significant difference in the water resistance of these bonds, with variations depending upon the specific primer used. The various proprietary catalysts that promote the reaction of the silane coupling agents are components that may affect the bond strength and water resistance. It is suggested that the use of catalysts in ceramic primers should be evaluated for the optimum performance of these products.

\section{Conclusion}

The clinical characteristics and durability of eight ceramic primers (seven commercial silane coupling agents and one alumina zirconia primer) were evaluated by measuring the wettability of a resin monomer and the bond strength between a resin composite and a glass surface.

(1) There was no significant decrease in tensile bond strength after thermal stress for MPS (control) relative to storage in water.

(2) No significant decrease in tensile bond strength after thermal stress was found in IN, MO, PL and RE commercial ceramic primers relative to water storage.

(3) There was no significant correlation between wettability and bond strength in the commercial ceramic primers.

These findings suggest that some commercially available ceramic primers might contribute to bond durability more than others.

\section{Acknowledgments}

This work was supported in part by JSPS KAKENHI Grant Number 23792300, 25462973 and 25463057.

\section{Conflict of Interest Statement}

The authors have no conflicts to report with respect to this work.

\section{References}

[1] Nishiyama, N., and Hayakawa, T. 1987. "Silane Coupling Agents." Adhes Dent 5: 129-33.

[2] Bouschlicher, M. R., Reinhardt, J. W., and Vargas, M. A. 1997. "Surface Treatment Techniques for Resin Composite Repair.” Am. J Dent. 10: 279-83.

[3] Chen, J. H., Matsumura, H., and Atsuta, M. 1998. "Effect of Etchant, Etching Period, and Silane Priming on Bond Strength to Porcelain of Composite Resin.” Oper Dent. 23: 250-7.

[4] Haga, M., and Sato, T. 1990. "Effects of Silane Coupling Agents and Adhesive Procedures of Porcelain Laminate Beneers Crown." Shika Journal 131: 283-8.

[5] Takahashi, H. 1987. "Clinical Evaluation of Silane Coupling Agents.” Shika Journal 25: 26-36.

[6] Takahashi, H. 1987. "Adhesion of Dental Ceramics." Adhes Dent 5: 135-44.

[7] Nihei, T., Kurata, S., Kondo, Y., Umemoto, K., Yoshino, N., and Teranaka, T. 2002. "Enhanced Hydrolytic Stability of Dental Composites by Use of Fluoroalkyltrimethoxysilanes." J. Dent Res. 81: 482-6.

[8] Ishida, H. 1981. "Molecular Aspects of Interfaces in Composite Materials." Koubunshi 30: 205-9.

[9] Kubo, S., Yoshitake, Y., Nakamura, N., Ohsawa, M., and Matsumoto, H. 1989. "Study on Wear of Composite Resins. Part 2. The Influence of Water Immersion on Wear of Composite Resins.” Jpn. J. Conserv. Dent. 32: 1311-30.

[10] Kubo, S., Oyama, K., Ohsawa, M., and Matsumoto, H. 1989. "Study on Wear of Composite Resins. Part 1. Subsurface Microstructure in Worn Layer of Composite Resins under Repeated Impact Loads.” Jpn. J. Conserv. Dent. 32: 1290-310.

[11] Ishida, S., and Nishiyama, N. 1989. "Study on Surface Treatment of Silica Filler: Hydrolytic Deterioration of the Silane Coupling Layer.” Jpn. J. Dent. Mater 8: 601-7. 
[12] Fukai, K., Nishiyama, N. 1992. "Study on a Silane Coupling Agent: Influence of an Acid on the Adsorption and Hydrolytic Stability of the Silane at the Interface." Jpn. J. Dent. Mater 11: 875-80.

[13] Nagashima, M., and Nishiyama, N. 1992. "Study on the Surface Treatment of Silica Filler: Synthesis and Hydrolytic Stability of Difunctional Silane.” Jpn. J. Dent. Mater 11: 415-22.

[14] Debnath, S., Wunder, S. L., McCool, J. I., and Baran, G. R. 2003. "Silane Treatment Effects on Glass/Resin Interfacial Shear Strengths." Dent. Mater 19: 441-8.

[15] Ozcan, M., Matinlinna, J. P., Vallittu, P. K., Huysmans, and M. C. 2004. "Effect of Drying Time of 3-Methacryloxypropyltrimethoxysilane on the Shear Bond Strength of a Composite Resin to Silica-Coated Base/Noble Alloys." Dent. Mater 20: 586-90.

[16] Nihei, T., Kurata, S., Kondoh, Y., Yoshino, N., and Teranaka, T. 2000. "Synthesis of Methacrylate Silane Coupling Agents with Fluoroalkyl Group at the Side-Chain and the Effect of Surface Treatment on a Glass Plate.” Jpn. J. Dent. Mater 19: 509-18.

[17] Ohashi, K., Nihei, T., Kurata, S., Kondoh, Y., Umemoto, K., Yoshino, N., and Teranaka, T. 2005. "Adhesion of Resin to Ceramic Surfaces Modified with Phenyl Group Silane Coupling Agents Containing a Double Bond.” Jpn.
J. Dent. Mater 24: 247-52.

[18] Nihei, T., Kurata, S., Ohashi, K., Kondoh, Y., Umemoto, K., Yoshino, N., and Teranaka, T. "Water Resistance of Novel Silane Coupling Agents Having Aromatic Group Substituted a Reactive Double Bond." Jpn. J. Dent. Mater 24: 1-8.

[19] Ohashi, K., Nihei, T., Mori, R., Kurata, S., Umemoto, K., and Teranaka, T. 2009. "Modified Effect of Adding Catalyst with Silane Coupling Treatment." Jpn. J. Conserv. Dent. 52: 161-7.

[20] Aida, M., Gotou, H., Nishiyama, N., Omura, Y., Wakami, M., Watanabe, T., and Sakurada, T. 2006. "Storage Effect of Ceramic Primer on Bond Bonding Efficacy of Resin." Adhes Dent. 24: 190-5.

[21] Yamaguchi, S. 1975. Kagakusousetsu No. 8 Fukugouzairyou. 1st ed. Tokyo: Gakkaisyuppan Center, 200-9.

[22] Meng, X., Yoshida, K., Taira, Y., Kamada, K., and Luo, X. 2011. "Effect of Siloxane Quantity and $\mathrm{pH}$ of Silane Coupling Agents and Contact Angle of Resin Bonding Agent on Bond Durability of Resin Cements to Machinable Ceramic.” J. Adhes Dent. 13: 71-8.

[23] Anagnostopoulos, T., Eliades, G., and Palaghias, G. 1993. "Composition, Reactivity and Surface Interactions of Three Dental Silane Primers.” Dent. Mater 9: 182-90. 